\title{
Comportamento e ciclo de vida de Epilachna vigintioctopunctata (Fabricius) (Coleoptera, Coccinellidae) em Lycopersicum esculentum Mill. (Solanaceae) ${ }^{1}$
}

\author{
Marileusa Araujo-Siqueira ${ }^{2} \&$ Lúcia Massutti de Almeida ${ }^{2}$ \\ ${ }^{1}$ Contribuição número 1481 do Departamento de Zoologia, Universidade Federal do Paraná. \\ 2 Departamento de Zoologia, Universidade Federal do Paraná. Caixa Postal 19020, 81531-980 Curitiba, Paraná, Brasil. \\ Bolsista do CNPq. E-mail: marileusa@yahoo.com.br; lalmeida@ufpr.br
}

\begin{abstract}
Behavior and life cycle of Epilachna vigintioctopunctata (Fabricius) (Coleoptera, Coccinellidae) in Lycopersicum esculentum Mill. (Solanaceae). Epilachna vigintioctopunctata (Fabricius, 1775) is a pest of many plants of agricultural importance in Asia. In 1990 the species was found in Brazil, near Curitiba, State of Paraná, in cucurbitaceous and in February of 2002 specimens were collected in Solanum americanum Mill. (Solanaceae) in Itajaí, State of Santa Catarina. Adults were kept under $\pm 24^{\circ} \mathrm{C}, \geqslant 53 \%$ of relative humidity, with 12 hours of photoperiod, and fed with leaves of Lycopersicum esculentum Mill. (Solanaceae). The average was 24.45 eggs per oviposition and incubation period was 7.14 days. The viability of the eggs was $63.68 \%$. The average of $\mathrm{st}^{\mathrm{st}}, 2^{\text {nd }}, 3^{\text {rd }}$ and $4^{\text {th }}$ larval instars was $5.88 ; 4.62 ; 5.88$ and 9.81 days, respectively. The average of the larval development was 26.19 days and the pupal 8.19 days. The average of life cycle was 41.44 days, and of the period of pre-oviposition, oviposition and pos-oviposition was $23.57,17.86$ e 53.86 days, respectively and the fecundity of the females was 59.78 eggs. The average of longevity of male and female was 81.44 and 97.89 days, respectively.
\end{abstract}

KEY WORDS. Biology, Epilachninae, tomato plant.

RESUMO. Epilachna vigintioctopunctata (Fabricius, 1775) é praga de diversas plantas de importância agrícola na Ásia. Em 1990 foi encontrada no Brasil, em cucurbitácea próximo a Curitiba, Estado do Paraná. Em fevereiro de 2002, foram coletados em Solanum americanum Mill. (Solanaceae) (maria-preta) em Itajaí, Estado de Santa Catarina. Adultos foram mantidos em $\pm 24^{\circ} \mathrm{C}$, umidade relativa $\geqslant 53 \%, 12 \mathrm{~h}$ fotofase e alimentados com folhas de Lycopersicum esculentum Mill. (Solanaceae). A média foi de 24,45 ovos por postura e o período de incubação de 7,14 dias. A viabilidade dos ovos foi de $63,68 \%$. A média do $1^{\circ}, 2^{\circ}, 3^{\circ}$ e $4^{\circ}$ instares larvais foi em média 5,$88 ; 4,62$; 5,88 e 9,81 dias, respectivamente. $O$ período larval totalizou em média 26,19 dias e o pupal 8,19 dias. $O$ ciclo de vida durou em média 41,44 dias. $O$ período médio de pré-oviposição, oviposição e pós-oviposição foi respectivamente de $23,57,17,86$ e 53,86 dias e a fecundidade das fêmeas de 59,78 ovos. A longevidade média do macho e da fêmea foi 81,44 e 97,89 dias, respectivamente.

PALAVRAS CHAVE. Biologia, Epilachninae, tomateiro.

O gênero Epilachna Chevrolat, 1837 (Epilachninae) apresenta mais de 180 espécies no hemisfério ocidental; alimentam-se de folhas de vegetais, principalmente de Cucurbitaceae e Solanaceae (HAYWARD 1942, Bose 1943, Silva et al. 1968, GoRdON 1976, Ali \& El SAeAdy 1981).

$\mathrm{Na}$ região sul do Brasil são relativamente comuns Epilachna paenulata (Germar, 1824), E. spreta (Mulsant, 1850) e E. cacica (Guérin, 1844), alimentando-se de Cucurbita pepo L. (Cucurbitaceae) (aboboreira), C. maxima Duchesne, C. moschata Duchesne, Lagenaria vulgaris Ser., além de folhas de Sechium edule (Jacq.) Sw. (chuchuzeiro) e de Cucumis sativus L. (pepineiro). Epilachna clandestina Mulsant, 1850 alimenta-se de Cayaponia ficifolia (Chambers) Cogn. e Cucurbita pepo (FonseCA \& Autuori 1931, Precetti et al. 1977, Silva et al. 1968, Almeida \&
Marinoni 1986, Almeida \& Ribeiro 1986, Marinoni \& Ribeiro 1987, Ribeiro \& Almeida 1989, Marinoni \& Giambarresi 1992).

Epilachna vigintioctopunctata (Fabricius, 1775) é uma praga séria de algumas plantas de importância agrícola na Ásia, incluindo batata, beringela, tomate, tabaco, abóbora, pepino, abobrinha, amendoim, melancia, alfafa, algodão e banana (SCHAEFER 1983).

Alguns experimentos biológicos têm sido conduzidos com esta espécie. Atwal \& SETHI (1977) trabalharam com dados biológicos obtidos com diferentes temperaturas e umidade no desenvolvimento e sobrevivência; a dinâmica populacional da espécie foi estudada por NAKAMURA et al. (1988) em Sumatra, utilizando como alimento folhas de berinjela. RAJAGOPAL \& TRIVEDI (1989) observaram a ocorrência de predadores, parasi- 
tóides, fungos e bacilos, além de utilizarem inseticidas, visando o controle de E. vigintioctopunctata, praga de folhas de batata na Índia.

Esta espécie foi encontrada pela primeira vez no sul do Brasil, na rodovia BR 101, próximo a Curitiba, em outubro de 1990, em uma cucurbitácea não identificada. Em 1991, foi coletada em Paranaguá, em Piper nigrum L. (pimenta) e em 1992, em Solanum americanum Mill. (maria-preta), em Itajaí (SCHroder et al. 1993).

Estudou-se o comportamento e ciclo de vida de Epilachna vigintioctopunctata, enfocando principalmente os aspectos de viabilidade, duração dos estágios, sobrevivência e fecundidade em condições de laboratório, utilizando-se como planta hospedeira Lycopersicum esculentum Mill. (Solanaceae) (tomateiro).

\section{MATERIAL E MÉTODOS}

Os bioensaios foram conduzidos no Laboratório de Sistemática e Bioecologia de Coleoptera, Departamento de Zoologia, Universidade Federal do Paraná, Curitiba, à temperatura média de $\pm 24^{\circ} \mathrm{C}$, umidade relativa de $\geqslant 53 \%$ e 12 horas de fotofase.

Adultos e larvas de Epilachna vigintioctopunctata foram coletados em folhas de Solanum americanum Mill. (maria-preta) em Itajaí, Santa Catarina, em fevereiro de 2002. Os insetos adultos obtidos a partir das posturas de fêmeas coletadas no campo foram criados em recipientes plásticos semitransparentes, com capacidade para $500 \mathrm{ml}$. Devido à dificuldade de se encontrar a planta hospedeira foram testadas algumas espécies alternativas de Cucurbitaceae e Solanaceae e a de Lycopersicum esculentum Mill. (Solanaceae) (tomateiro) foi a que teve melhor aceitação. $\mathrm{O}$ alimento era fornecido diariamente com a troca de recipiente, quando necessário, até a obtenção dos casais.

Os exemplares foram separados aos pares com base no tamanho, pois geralmente os machos são menores que as fêmeas.

Foram utilizados nove casais para obtenção das posturas, as quais foram separadas em placas-de-petri forrados com papel filtro umedecido em água destilada. Em cada placa foram colocadas de duas a três folhas de tomateiro como alimento. A partir do $3^{\circ}$ instar, em cada placa foram mantidas no máximo 15 larvas, para não haver falta de alimento e, conseqüentemente, canibalismo.

Os casais 7, 8 e 9 deram origem a indivíduos que sobreviveram apenas até o $1^{\circ}$ instar, por esse motivo os mesmos foram considerados apenas quanto à viabilidade dos ovos e longevidade dos adultos.

Foram calculadas as médias com os respectivos erros, de acordo com o número de insetos obtidos em cada fase de desenvolvimento, utilizando-se a estatística descritiva de ANOVA.

\section{RESULTADOS E DISCUSSÃO \\ Comportamento}

Os ovos de E. vigintioctopunctata são alongados e apresentam coloração amarelada (Fig. 1). As posturas foram observadas, principalmente, na parte abaxial das folhas de tomatei- ro ou na região interna dos recipientes, e, mais raramente, no fundo dos mesmos. Epilachna cacica e E. spreta, criadas nas mesmas condições, fazem as posturas sob as folhas de aboboreira, enquanto que E. paenulata, nas paredes do recipiente (ALMEIDA \& MARINONI 1986).

Os ovos foram postos em grupos e geralmente dispostos em uma única camada (Fig. 1), porém, algumas posturas apresentaram sobreposição tornando a massa de ovos bastante densa, com duas a três camadas, uma sobre as outras. NAKAMURA et al. (1984) relatam que o tamanho do recipiente utilizado para a criação em laboratório de duas espécies de Henosepilachna (Li, 1961), tratadas como H. sparsa (Herbst, 1786) "espécie C" e "espécie D", alimentadas com cucurbitáceas, influenciou o tamanho da massa de ovos. Entretanto, Richards \& Filewood (1988) verificaram que o substrato influencia o tamanho da postura das espécies de Epilachna.

Logo após a eclosão, as larvas permaneciam próximas à massa de ovos por aproximadamente um dia alimentando-se das exúvias ou dos ovos inviáveis.

Não foi observado canibalismo entre as larvas, entretanto duas pupas foram predadas por larvas de último instar. EDONA \& SoAns (1971) relatam que em alguns estágios de desenvolvimento pode ocorrer uma tendência ao canibalismo, mesmo que folhas frescas do alimento estejam disponíveis. Em Henosepilachna sparsa, adultos e larvas ocasionalmente se alimentam de ovos, sendo que alguns adultos podem consumir 11 ovos em cerca de 30 minutos. Geralmente as fêmeas preferem consumir ovos postos por outras espécies de besouros, quando disponíveis.

Com relação ao processo de muda, as larvas diminuíam suas atividades físicas e metabólicas para realizarem a ecdise. A coloração inicial após a troca era amarela brilhante, semelhante ao ovo, tornando-se em seguida amarelada com placas castanhas, de onde saem os escolos (Fig. 2). No final do $4^{\circ}$ instar a larva se encurva e se fixa no substrato, passando para o estágio de pupa, permanecendo com a exúvia do último instar aderida na região posterior do corpo (Fig. 3).

Epilachna vigintioctopunctata (Fig. 4) alimenta-se do parênquima das folhas de tomateiro demarcando-as com as mandíbulas. Este comportamento também foi observado em E. varivestis (Mulsant, 1850) (HowARD 1936), Epilachna clandestina e E. borealis (Fabricius, 1775) (Fonseca \& AUtUori 1931, Brannon 1937), E. paenulata, E. cacica e E. spreta (Almeida \& MARINONI 1986).

\section{Postura}

As posturas apresentaram um total médio de 24,45 $\pm 2,35$ ovos, variando entre 4 e 47 ovos (Tab. I). O número médio de ovos por postura foi próximo ao observado em E. clandestina (MARINoni \& GiambarResi 1992) e inferiores aos obtidos em $E$. paenulata à temperatura de $20 \pm 0,5^{\circ} \mathrm{C}$ (GANHO \& MARINONI 2000).

Foram realizadas em média $2,44 \pm 0,55$ posturas (Tab. 1 ) totalizando 538 ovos, dos quais 321 viáveis. Este resultado foi menor que os observados por GanHo \& MARINONI 2000 para $E$. paenulata e por MARINONI \& Giambarresi 1992 para E. clandesti- 

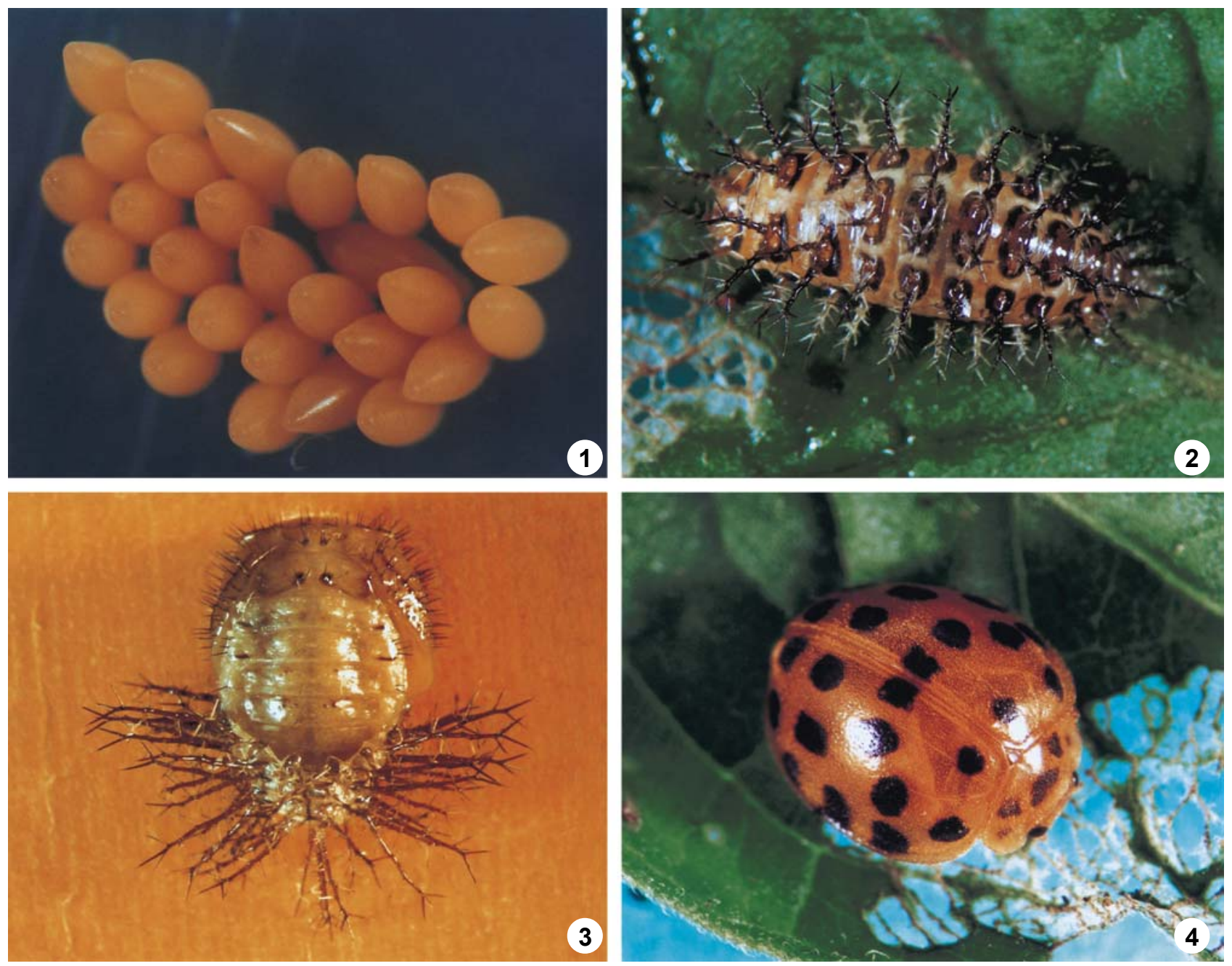

Figuras 1-4. Epilachna vigintioctopunctata: (1) postura; (2) larva de $4^{\circ}$ instar; (3) pupa; (4) adulto.

na, que foram respectivamente 12,5 e 8 posturas, em média por fêmea.

O período médio de incubação foi de 7,14 $\pm 0,23$ dias e variou de 5 a 9 dias (Tab. I). Rajagopal \& Trivedi (1989) indicaram uma variação de 3 a 5 dias para a eclosão do ovo de $E$. vigintioctopunctata. ALMEIDA \& MARINONI (1986) registraram períodos de incubação superiores para Epilachna paenulata (8 dias), E. cacica (10,7 dias) e E. spreta (13,7 dias).

A viabilidade de ovos foi em média $63,68 \%$, variando de $26,2 \%$ até $93,1 \%$. O número médio de ovos viáveis foi de 15,09 $\pm 1,86$ ovos, variando de 3 a 40 ovos (Tab. I). O valor obtido foi superior aos encontrados para E. clandestina (53\%) e E. paenulata (49\%) e muito maior que o de $E$. cacica $(8,7 \%)$.

A taxa de mortalidade, de $36,32 \%$, pode estar associada a fatores abióticos como fotoperíodo, temperatura e planta hospedeira. Além disso, em posturas com um grande número de ovos, observou-se que aqueles que permaneciam nas camadas inferiores da massa, geralmente eram inviáveis.

\section{Larva}

O desenvolvimento larval apresentou quatro instares e representou em média $63,2 \%$ do ciclo de vida. O $1^{\circ}$ instar durou em média, 5,88 $\pm 0,26$ dias, variando de 4 a 8 dias. $\mathrm{O} 2^{\circ}$ instar foi o período de desenvolvimento mais curto, tendo duração média de 4,62 $\pm 0,27$, variando de 3 a 6 dias. O $3^{\circ}$ instar variou de 4 a 8 dias, com duração média de 5,88 $\pm 0,33$. O $4^{\circ}$ instar foi o que apresentou maior duração no ciclo de vida, com média de 9,81 $\pm 0,48$ dias, variando de 7 a 14 dias (Tab. II).

Para Rajagopal \& TRivedi (1989), a duração dos quatro instares de $E$. vigintioctopunctata estudados foi: $1^{\circ}$ instar (4-6 dias); $2^{\circ}$ instar (4-6 dias); $3^{\circ}$ instar (3-7 dias); $4^{\circ}$ instar (5-8 dias), números que estão próximos aos encontrados neste trabalho, havendo maior variação apenas no $4^{\circ}$ instar. 
Tabela I. Número de posturas, número médio de ovos por postura, mínimo e máximo por postura, duração média do período do ovo, número médio de ovos viáveis e viabilidade média dos ovos de $\mathrm{E}$. vigintioctopunctata, em condições de laboratório $\left( \pm 24{ }^{\circ} \mathrm{C}, \mathrm{UR} \geqslant 53 \%\right.$, $12 \mathrm{~h}$ fotofase).

\begin{tabular}{ccccccc}
\hline \hline Repetições & No de posturas & No de ovos/postura & $\begin{array}{c}\text { No de ovos mín. } \\
\text { máx./ postura }\end{array}$ & $\begin{array}{c}\text { Duração (dias) } \\
\text { No de ovos viáveis }\end{array}$ & Viabilidade (\%) \\
\hline 1 & 1 & 42,0 & - & 6,0 & 11,0 & 26,2 \\
3 & 5 & 24,2 & $10-35$ & 6,8 & 16,8 & 70,8 \\
4 & 5 & 25,0 & $4-47$ & 7,2 & 13,0 & 61,5 \\
5 & 3 & 18,3 & $12-25$ & 7,7 & 9,0 & 46,0 \\
6 & 1 & 25,0 & - & 7,0 & 7,0 & 28,0 \\
7 & 2 & 17,0 & $11-23$ & 6,0 & 11,0 & 69,1 \\
8 & 3 & 27,0 & $11-44$ & 7,3 & 24,0 & 83,5 \\
9 & 1 & 29,0 & - & 9,0 & 27,0 & 93,1 \\
Variação & 1 & 26,0 & - & 8,0 & 17,0 & 65,4 \\
\hline \hline
\end{tabular}

(-) Os casais 1, 5, 8 e 9 só realizaram uma postura, por isso o número máximo e mínimo de ovos não foram considerados.

Tabela II. Médias ( $\Psi \mathrm{P})$ da duração do período larval e dos quatro instares larvais de E. vigintioctopunctata, em condições de laboratório ( $\pm 24 \circ \mathrm{C}, \mathrm{UR} \geqslant 53 \%, 12 \mathrm{~h}$ fotofase).

\begin{tabular}{|c|c|c|c|c|c|}
\hline \multirow{2}{*}{ Repetições } & \multicolumn{4}{|c|}{ Duração (dias) } & \multirow{2}{*}{ Total } \\
\hline & 10 instar & 20 instar & 3o instar & 4 o instar & \\
\hline $\bar{~} 1$ & 5,0 & 3,0 & 4,0 & 9,0 & 21,0 \\
\hline 2 & 6,0 & 4,0 & 5,5 & 10,0 & 25,5 \\
\hline 3 & 6,8 & 5,0 & 6,0 & 10,2 & 28,0 \\
\hline 4 & 5,7 & 5,7 & 5,7 & 10,7 & 27,7 \\
\hline 5 & 5,0 & 4,0 & 8,0 & 8,0 & 25,0 \\
\hline 6 & 4,5 & 4,5 & 6,5 & 8,5 & 24,0 \\
\hline$\overline{x \pm E}$ & "5,88 $\pm 0,26$ & "4,62 $\pm 0,27$ & 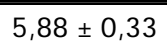 & $9,81 \pm 0,48$ & $26,19 \pm 0,73$ \\
\hline Variação & $4-8$ & $3-6$ & $4-8$ & $7-14$ & $21-31$ \\
\hline
\end{tabular}

Para E. spreta o 1ㅇinstar foi o período de menor duração (Almeida \& M ARINONI 1986) diferindo dos presentes resultados, onde o 2 instar foi o menor.

Quando observado o tempo de duração de cada um dos estágios de desenvolvimento, nota-se que os instares intermediários ( $2^{\circ}$ e 3으) são os mais curtos, enquanto o estágio de ovo eo de pupa são os mais longos. Em E. varivestis também seobserva menor duração dos instares intermediários (M cavor \& SMITH 1979).

O período larval totalizou em média $26,19 \pm 0,73$ dias e apresentou uma variação de 21 a 31 dias (Tab. II ), menor vari- ação que os 17 a 27 dias registrados por RajAgopal \& TRIVEDI (1989) para a mesma espécie. O resultado foi intermediário ao encontrado em E. paenulata (20,8 dias) eE. spreta (30,3 dias) e semelhante ao obtido para E. cacica (26 dias) por Almeida \& MARINONI (1986).

Com relação à sobrevivência média dos quatro instares, foram obtidos os seguintes resultados: 10 instar, $54,70 \% \pm 6,48$; 2o instar, 79,74\% $\pm 6,88$; 3o instar, $84,67 \% \pm 11,07 ; 40$ instar, $94,78 \% \pm 4,79$ (Tab. III). Estes dados foram menores que os encontrados por M ARINoNI \& Ribero (1987) para E. paenulata, sendo queas mai ores diferenças ocorreram no 1 ㅇinstar (84,3\%), 
Tabela. III. Médias (士EP) do número de ovos por postura, da sobrevivência do período larval e dos instares larvais de E. vigintioctopunctata, em condições de laboratório ( $\pm 24{ }^{\circ} \mathrm{C}, \mathrm{UR} \geqslant 53 \%, 12 \mathrm{~h}$ fotofase).

\begin{tabular}{|c|c|c|c|c|c|c|}
\hline \multirow{2}{*}{ Repetições } & \multirow{2}{*}{ № de ovos/postura } & \multicolumn{4}{|c|}{ "Sobrevivência \% } & \multirow{2}{*}{ Total } \\
\hline & & 10 instar & 2 o instar & 3o instar & 40 instar & \\
\hline 1 & 42,0 & 63,64 & 100 & 100 & 100 & 63,6 \\
\hline 2 & 24,2 & 53,54 & 73,79 & 74 & 70,91 & 50,6 \\
\hline 3 & 25,0 & 24,93 & 78,00 & 100 & 97,78 & 17,4 \\
\hline 4 & 18,3 & 57,80 & 66,67 & 100 & 100 & 46,6 \\
\hline 5 & 25,0 & 71,43 & 60,00 & 33,33 & 100 & 14,3 \\
\hline 6 & 17,0 & 56,84 & 100 & 100 & 100 & 56,8 \\
\hline$x \pm E P$ & $23,65 \pm 2,68$ & $54,70 \pm 6,48$ & $79,74 \pm 6,88$ & $84,67 \pm 11,07$ & $94,78 \pm 4,79$ & $38,79 \pm 6,74$ \\
\hline
\end{tabular}

seguida dos 20 (89,8\%) e 3o $(95,7 \%)$ instares; no 4ㅇinstar $(95,1 \%)$ a sobrevivência foi muito semelhante.

A sobrevivência larval média foi de $38,79 \%$ (Tab. III) variando de $7,10 \%$ a $100,0 \%$, sendo menor que os val ores registrados em E. paenulata $(52,8 \%)$ e em E. cacica $(52,2 \%)$ (PrEcETा। et al. 1977).

Não foi possível diagnosticar a causa responsável pela mortalidade das larvas dos casais 7,8 e 9 , visto que as posturas tinham aparência saudável ecerca de $81 \%$ dosovos foram viáveis.

Pupa

O estágio de pupa (Fig. 3) teve duração média de 8,19 \pm 0,36 dias e apresentou uma variação de 6 a 11 dias (Tab. IV). Para a mesma espécie houve uma variação de 5 a 9 dias (RAJAgopal \& Trivedi 1989). Almeida \& Marinoni (1986) obtiveram para E. paenulata duração muito semel hante (8 dias), enquanto que paraE. spreta ( 9,7 dias) eE. cacica ( 9 dias) o período foi um pouco superior.

A sobrevivência média do estágio pupal foi alta, 98,71\% (Tab. IV), superior ao encontrado para E. cacica, de $83,3 \%$ (Precerti et al. 1977).

Tabela IV. Número de pupas e médias (\#P) da duração e sobrevivência do período pupal de E. vigintioctopunctata, em condições de laboratório ( $\pm 244^{\circ} \mathrm{C}, \mathrm{UR} \geqslant 53 \%, 12 \mathrm{~h}$ fotofase).

\begin{tabular}{cccc}
\hline \hline Repetições & № de pupas & Duração (dias) & Sobrevivência (\%) \\
\hline \hline 1 & 7 & 6,0 & 100 \\
2 & 37 & 7,0 & 94,4 \\
3 & 12 & 8,6 & 100 \\
4 & 8 & 9,7 & 100 \\
5 & 1 & 8,0 & 100 \\
6 & 13 & 8,5 & 100 \\
\hline X士EP & 13 & $8,19 \pm 0,36$ & $98,71 \pm 1,39$ \\
Variação & $1-37$ & $6-11$ & $77,8-100$ \\
\hline
\end{tabular}

\section{Ciclo de vida}

O ciclo de vida deE. vigintioctopunctata, ou seja, o período compreen di do entre a postura atéa emergência do adulto, teve a duração média de 41,44 $\pm 0,82$ dias, variando de 33 a 50 dias (Tab. V). RAJAG OPAL \& TRIVEDI (1989) encontraram um intervalo maior para a mesma espécie (25 a 45 dias). Em estudos realizados por ALmeIDA \& MARINoNi (1986), E. paenulata foi a espécie com menor tempo de duração do ciclo de vida (36,8 dias), seguida por E. cacica (46 dias) eE. spreta (53,7 dias).

Tabela. V. Médias ( $\pm E P$ ) da duração do ciclo de vida deE. vigintioctopunctata, em condições de laboratório $\left( \pm 24{ }^{\circ} \mathrm{C}, \mathrm{UR} \geqslant 53 \%, 12\right.$ $\mathrm{h}$ fotofase).

\begin{tabular}{cc}
\hline Repetições & Duração (dias) \\
\hline \hline 1 & 33,0 \\
2 & 39,3 \\
3 & 43,8 \\
4 & 45,1 \\
5 & 40,0 \\
6 & 38,5 \\
\hline X \pm EP & $41,44 \pm 0,82$ \\
Variação & $33-50$ \\
\hline
\end{tabular}

Períodos de pré-oviposição, oviposição e pósoviposição A duração média do período depré-oviposição, quecompreen de da emergência da fêmea até a primeira postura, foi de 23,57 $\pm 4,43$ dias, variando de 17 a 52 dias (Tab. VI).

Estes valores foram inferiores aos obtidos por GANHO \& M ARINONI (2000) ao estudarem E. paenulata, que foi em média de 71,40 dias, com variação de 37 a 113 dias e também menor que o verificado em E. clandestina, 50,33 dias MARINONI \& GiambarResI 1992). 
Tabela VI. Períodos de pré-oviposição, oviposição, pós-oviposição e fecundidade de E. vigintioctopunctata, em condições de laboratório ( $\pm 24 \circ \mathrm{C}$, $, U R \geqslant 53 \%, 12 \mathrm{~h}$ fotofase).

\begin{tabular}{ccccc}
\hline \hline \multirow{2}{*}{ Fêmeas } & \multicolumn{3}{c}{ Período (dias) } \\
\cline { 2 - 5 } & Pré-oviposição & Oviposição & Pós-oviposição & Fecundidade \\
\hline \hline & 20 & 1 & 114 & 42 \\
3 & 19 & 25 & 42 & 121 \\
4 & 33 & 28 & 30 & 125 \\
5 & 17 & 13 & 81 & 55 \\
6 & 17 & 1 & 29 & 25 \\
7 & 31 & 26 & 38 & 34 \\
8 & 28 & 31 & 43 & 81 \\
9 & 52 & 1 & 46 & 29 \\
X \pm EP & 49 & 1 & 65 & 26 \\
Variação & $23,57 \pm 4,43$ & $17,86 \pm 4,45$ & $53,86 \pm 9,31$ & $59,78 \pm 13,31$ \\
\hline
\end{tabular}

O período médio de oviposição, compreendido entre a primeira e a última postura, foi de $17,86 \pm 4,45$ dias, variando de 1 a 31 dias (Tab. VI). Estes valores foram baixos, comparando-se aos obtidos por GANHO \& M ARINONI (2000) paraE. paenulata, (47,60 dias) e ainda menor que em E. clandestina (75,5 dias).

O período de pós-oviposição, abrangendo o intervalo entre a última postura até a morte da fêmea, foi em média de $53,86 \pm 9,31$ dias, variando de 29 a 114 dias (Tab. VI), resultado inferior ao registrado por M ARINONI \& G IAMBARRESI (1992) paraE. clandestina (70,2 dias).

\section{Fecundidade}

A fecundidade das fêmeas deE. vigintioctopunctata foi em média de $59,78 \pm 13,31$ ovos, apresentando uma grande variação, de 25 a 125 ovos por fêmea (Tab. VI); este resultado foi muito inferior ao obtido com E. cacica por PRECETTI et al. (1977) (437,1 ovos por fêmea), E. paenulata ( 555 ovos) (GANHo \& M ARINONI 2000), E. clandestina (216 ovos) (M ARINONI \& GIAMBarResI 1992) e E. chrysomelina (Fabricius, 1775) $(308,3)$ (ALI \& EL-SAEADY 1981).

A baixa taxa defecundidade encontrada pode estar relacionada à nutrição, já queo al imento queo inseto estava utilizando no campo, maria-preta (Solanum americanum) foi substituído por folha de tomateiro (Lycopersicum escul entum), ambos da família Solanaceae. Segundo Stewart et al. (1991), a reprodução depende diretamente da quantidade equalidade do alimento assimilado pela espécie; além disso, a quantidade de ovos depositados é influenciada pelo investimento de energia gasta durantea reprodução.

\section{Longevidade dos adultos}

A longevidade do macho foi em média de $81,44 \pm 10,26$ dias, oscilando de 40 até 135 dias (Tab. VII). RAJAGopal \& TRIVEDI (1989) encontraram um intervalo maior (11 a 403 dias) para a mesma espécie. Nas fêmeas, a longevidade foi em média 97,89 \pm 8,03 dias, com variação de 47 a 135 dias (Tab. VII), sendo inferior aos valores médios verificados em E. cacica (104 dias) (PRECETII et al. 1977) eE. paenulata (138 dias) (GANHO \& M ARINONI 2000).

Em Coccinellidae, a longevidade pode variar muito. Algumas espécies apresentam longevidade média muito maior nas fêmeas que nos machos, como o observado neste trabal ho. No entanto, há registros em que fêmeas que convivem com machos morrem antes, possivelmente em função da perda de energia durante a oviposição; fêmeas não pareadas chegam a viver $20 \%$ a $30 \%$ a mais (M ATSUKA et al. 1982).

Tabela VII. Longevidade, em dias, de macho e fêmea de E. vigintioctopunctata, em condições de laboratório $\left( \pm 24^{\circ} \mathrm{C}\right.$, UR $\geqslant$ $53 \%, 12 \mathrm{~h}$ fotofase).

\begin{tabular}{ccc}
\hline \hline Repetições & Macho & Fêmea \\
\hline \hline 1 & 77 & 135 \\
2 & 79 & 86 \\
3 & 135 & 91 \\
4 & 96 & 111 \\
5 & 43 & 47 \\
6 & 40 & 95 \\
7 & 99 & 102 \\
8 & 60 & 99 \\
9 & 104 & 115 \\
\hline \hline X \pm 甲 & $81,44 \pm 10,26$ & $97,89 \pm 8,03$ \\
Variação & $40-135$ & $47-135$ \\
\hline
\end{tabular}




\section{A GRA DECIMENTOS}

Agradecemos ao pesquisador Luciano de A. Moura (Museu de Ciências Naturais, Fundação Zoobotânica do Rio Grande do Sul), pela coleta dos insetos, ao Consel ho Nacional de Desenvolvimento Cientifico eTecnológico (CNPq), pelo auxílio concedido e bolsas de Iniciação Científica, M estrado e Produtividade em Pesquisa.

\section{REFERÊN CIA S BIBLIO GRÁ FICAS}

ALI, M.A. \& A.A. El SAEADY. 1981. Influence of temperature, photoperiod and host plant on the bionomics of the melon Iadybird Epilachna chrysomelina (Coleoptera: Coccinellidae). Zeitsch rift für angewandte Entomologie, Hamburg, 91 (3): 256-262.

AlmeidA, L.M. \& R.C. M ARINoni. 1986. Desenvolvimento de três espécies de Epilachna (Coleoptera: Coccinellidae) em três combinações de temperatura e fotoperíodo. Pesquisa Agropecuária Brasil ei ra, Brasília, 21 (9): 927-939.

Almeida, L.M. \& C.S. Ribelro. 1986. Morfologia dos estágios imaturos de Epilachna cacica Guérin, 1844 (Coleoptera: Coccinellidae). Revista Brasileira de Entomologia, São PauIo, 30 (1): 43-49.

Atwal, A.S. \& S.L. Sethi. 1977. Influence of different levels of temperature and relative humidity on the speed of development and survival of Epilachna vigintioctopunctata F. (Coleoptera: Coccinellidae). Indian Journal of Ecology, Ludhiana, 4 (1): 91-93.

BosQ, J.M. 1943. Segunda lista de coleópteros de la República Argentina, dañinos a la agricultura. IngenieriaAgronomica, Caracas, 4: 5-79.

Brannon, L.W. 1937. Life-history studies of the squash beetle in Alabama. Annals of the Entomological Society of America, Lanham, 30: 43-50.

Edona, V.I. \& A.B. Soans. 1971. Cannibalism in the Epilachna beetles, Henosepilachna sparsa Herbst. Journal of the Bombay Natural History Society, Bombay, 68: 479.

FonseCA, J.P. \& M. Autuorı. 1931. Contribuição para a biologia de Solanophila clandestina (Mulsant) (Coccinellidae: Coleoptera). Revista deEntomologia, Rio de Janeiro, 1 (2): 219224.

Ganho, N.G. \& R.C. MaRinoni. 2000. Algumas características da reprodução e ontogênese de Epilachna paenulata (Germar) (Coleoptera, Coccinellidae, Epilachninae). Revista Brasileira deZoologia, Curitiba, 17 (2): 445-454.

Gordon, R.D. 1976. A revision of the Epilachninae of the Western Hemi sphere (Coleoptera: Coccinellidae). Wa shington, ARS, USDA, Technical Bulletin No. 1493, 409p.

HaYward, K.J. 1942. Primera lista de insectos Tucumanos perjudiciales. Tucumán. Estación Experimental Agro-Industrial "Obispo Colombres" Publicación Miscel anea, San Miquel deTucumán: 1-110.

HowARD, M.F. 1936. Feeding of the Mexican bean beetle larva.
U.S. Department of Agriculture Farmers' Bulletin, Washington, 1624: 1-3.

Marinoni, R.C. \& C.S. Ribelro. 1987. Aspectos bionômicos de Epilachna paenulata (Germar, 1824) (Coleoptera: CoccineIlidae) em quatro diferentes plantas hospedeiras (Cucurbitaceae). Revista Brasilei ra de Entomologia, São Paulo, 31 (3): 421-430.

Marinoni, R.C. \& N. GiambarresI. 1992. Sobre a oviposição e ontogenia deEpilachna clandestina (Mulsant, 1850) (Coleoptera: Coccinellidae). Revista Brasileira de Entomologia, São Paulo, 36 (3): 535-540.

Matsuka, M.; M. Watanabe \& K. Niııma. 1982. Longevity and oviposition of Vedalia beetles on artificial diets. Environmental Entomology, Lanham, 11: 816-819.

M cavor, T.J. \& J.C. Smith. 1979. Feeding and developmental rates of the Mexican bean beetle on soybeans. Journal of Economic Entomology , Lanham, 72: 835-836.

NAKAMURA, K.; I. A BBAS \& A. HASYIM . 1984. Survivorship and fertility schedules of two epilachnine "species" feeding on curcubitaceous plants under laboratory conditions (Coleoptera: Coccinellidae). Applied Entomology and Zoology, Ibaraki, 19 (1): 59-66.

. 1988. Population dynamics of the phitophagous lady beetle, Epilachna vigintioctopunctata, in an egg plant field in Sumatra. Researcheson Population Ecology ,Tokyo, 30: 25-41.

Precetti, A.A.C.M.; J. Milanez; J.R.P. Parra \& E. Berti-Filho. 1977. Biologia e prejuízos causados por Epilachna cacica (Guérin, 1842) em aboboreira (Cucurbita moschata Duchesne). Ecossi stema, Espírito Santo do Pinhal , 2: 23-27.

Rajagopal, D. \& T.P. Trivedi. 1989. Status, bioecology and management of Epilachna vigintioctopunctata (Fab.) (Coleoptera: Coccinellidae) on potato in India: a review. Tropical Pest M anagement, London, 35 (4): 410-413.

Ribeiro, C.S. \& L.M. AlmeidA. 1989. Descrição dos estágios imaturos de Epilachna spreta (Muls., 1850) (Coleoptera: Coccinellidae), com redescrição, comentários e chave para três outras espécies. Revista Brasil eira de Zoologia, Curitiba, 6 (1): 99-110.

Richards, A.M. \& L.W. Filewood. 1988. The effect of agricultural crops and weeds on the bionomics of the pest species comprising the Epilachna vigintioctopunctata complex (Col., Coccinellidae). Journal of Applied Entomological, Berlin, 105: 88-103.

Schaefer, P.W. 1983. Natural enemies and host plants of species in the Epilachninae (Coleoptera: Coccinellidae) - a world list. University of Delaware. Agricul tural Experiment Station University of VermontBulletin, Burlington, 445: 1-42.

Schroder, R.F.W.; M.M. Athanas \& C. Pavan. 1993. Epilachna vigintioctopunctata (Coleoptera: Coccinellidae), new record for Western Hemisphere, with a review of host plants. Entomological News, Philadelphia, 104 (2): 111-112.

Silva, A.G.; S.R. Gonçalves; D.M. Galvão; A.J.L. Gonçalves; J. 
Gomes; M.N. Silva \& L. Simonı. 1968. Quarto Catálogo dos In setos que vivem nas plantas do Brasil, seus parasitose predadores. Rio de Janeiro, Ministério da Agricultura, Laboratório Central de Patologia Vegetal, $1^{\circ}$ Tomo, 622p.
Stewart, L.A.; J. L. Hemptinme \& A.F.G. Dixon. 1991. Reproductive tactics of ladybird beetles: relationships between egg size, ovariole number and developmental time. Functional Ecology, Oxford, 5: 380-385.

Recebido em 08.VI.2004; aceito em 19.VII.2004. 